\title{
Growth monitoring with the British 1990 growth reference
}

T J Cole

\begin{abstract}
Aim-To provide a simple method for assessing centile change based on the British 1990 growth reference.

Statistical method-The change in SD score over a period of time has SD $\sqrt{2(1-r)}$, where $r$ is the correlation between the first and second SD score. This leads to an SD score for centile change.

Data-Annual height measurements from 2 to 9 years for 318 children from the French longitudinal growth study.

Results-The correlations between heights at different ages are higher for shorter measurement intervals and at older ages. The chance of a child's height centile falling one centile band width is correspondingly smaller for shorter measurement intervals and at older ages. An increase in height measurement error reduces the correlations and dramatically increases the chances of centile crossing. Conclusions-Quantitative height monitoring based on centile change is provided for whole year periods between 2 and 9 years of age. Effective monitoring requires the measurement error to be as small as possible.

(Arch Dis Child 1997;76:47-49)
\end{abstract}

Keywords: growth monitoring; measurement error; height; velocity.

Growth references are designed to assess single measurements of height and weight, but are often used for growth monitoring, that is to assess changes in height and/or weight centile over time. A recent $B M \mathcal{F}$ leader ${ }^{1}$ discussed growth monitoring using the British 1990 growth reference. ${ }^{2}$ Subsequently, questioners asked how to quantify height centile crossing ${ }^{3}$ or the discrepancy between weight and height centiles. ${ }^{4}$ The questioners suggested guidelines for identifying abnormality in such cases, but offered no evidence to justify them.

In fact centile crossing can be quantified very simply. The British 1990 growth reference is doubly useful here: it converts weight and height centiles to SD scores, which are Normally distributed with mean 0 and SD 1, and the centile curves on the chart are equally spaced in SD score terms, two thirds (that is 0.67 ) of an SD score apart. So a change in centile can be read off the chart as a fraction of the distance between adjacent centile curves (here called the centile band width) irrespective of which curves they are. This in turn calibrates the chart for the user, expressing the centile change in SD score terms.

\section{Statistical method}

Convert the two centiles to SD scores, say $\mathrm{SDS}_{1}$ and $\mathrm{SDS}_{2}$, and calculate the difference between them: $\Delta$ SDS $=\operatorname{SDS}_{2}-\mathrm{SDS}_{1}$. So if a child's height shifts from the median $\left(\mathrm{SDS}_{1}=\right.$ $0)$ to near the 2 nd centile $\left(\mathrm{SDS}_{2}=-2\right)$ then $\Delta$ SDS $=-2$. The two centiles would normally be height at different ages, or could alternatively be weight and height at the same age.

It can be shown that in the reference population:

Mean of $\triangle S D S=0$

$\mathrm{SD}$ of $\Delta \mathrm{SDS}=\sqrt{2(1-r)}$

where $r$ is the correlation between SDS $_{1}$ and $\mathrm{SDS}_{2}$. This allows $\triangle \mathrm{SDS}$, the observed change in centile, to be expressed as an SD score for centile change (for example height gain):

$\mathrm{SD}$ score for centile change $=\frac{\Delta \mathrm{SDS}-\mathrm{mean}(\Delta \mathrm{SDS})}{\operatorname{SD}(\Delta \mathrm{SDS})}=$

$$
=\frac{\Delta \operatorname{SDS}}{\sqrt{2(1-r)}}
$$

Data

The correlation $r$ depends on the two measurements and the ages when they are taken. Data from the French longitudinal growth study ${ }^{5}$ are used to calculate age-on-age correlations for height. See also Cole for one year correlations from the French study, ${ }^{6}$ and Bailey for six month and one year correlations from the Harpenden growth study. ${ }^{7}$ The correlation between weight and height at the same age is about 0.7 (and is essentially constant between 3 and 11 years of age). ${ }^{8}$

\section{Results}

Table 1 gives correlations for height at ages from 2 to 9 years (sexes averaged) for between 204 and 318 subjects from the French longitudinal growth study. The correlations are higher for shorter measurement intervals and at older ages. Knowing the correlation, selected centiles for $\triangle$ SDS can be calculated using formula (1), or an individual child's $\Delta$ SDS can be expressed as an SD score or centile using formula (2).

For example, assume that $\Delta$ SDS represents the change in height SD score from 5 to 6 years of age, where the correlation $r$ is 0.981 (table 1). So $\triangle$ SDS is a measure of height gain. From formula (1) the SD of $\Delta \mathrm{SDS}$ is $\sqrt{2(1-0.981)}=$ 0.19 height SD score units. The 2 nd centile for $\triangle S D S$ is at 2 SDs below the mean, that is at -0.38 height SD score units. The centile 
Table 1 Correlations between heights measured at different ages, based on data from the French longitudinal growth study (sexes averaged). ${ }^{5}$ The sample sizes for each correlation are between 204 and 318

\begin{tabular}{|c|c|c|c|c|c|c|c|c|}
\hline & \multicolumn{8}{|c|}{ Age (years) } \\
\hline & 2 & 3 & 4 & 5 & 6 & 7 & 8 & 9 \\
\hline \multicolumn{9}{|c|}{ Age (years) } \\
\hline 2 & 1 & & & & & & & \\
\hline 3 & 0.941 & 1 & & & & & & \\
\hline 4 & 0.888 & 0.958 & 1 & & & & & \\
\hline 5 & 0.860 & 0.934 & 0.975 & 1 & & & & \\
\hline 6 & 0.835 & 0.912 & 0.958 & 0.981 & 1 & & & \\
\hline 7 & 0.824 & 0.893 & 0.944 & 0.971 & 0.983 & 1 & & \\
\hline 8 & 0.800 & 0.875 & 0.926 & 0.957 & 0.970 & 0.986 & 1 & \\
\hline 9 & 0.772 & 0.857 & 0.907 & 0.939 & 0.954 & 0.975 & 0.988 & 1 \\
\hline
\end{tabular}

Table 2 Effect of height measurement error $\delta$ on the proportion of children falling one centile band width or more between 5 and 6 years of age. The research measurement error $\varepsilon$ in formula (3) is taken as $0.25 \mathrm{~cm}$, and the population height $S D \sigma 5 \mathrm{~cm}$

\begin{tabular}{|c|c|c|c|c|c|}
\hline & Value & & & & \\
\hline Measurement error $\delta(\mathrm{cm})$ & 0.25 & 0.4 & 0.6 & 0.8 & 1 \\
\hline Adjusted correlation $r^{\prime}(3)$ & 0.981 & 0.977 & 0.969 & 0.958 & 0.944 \\
\hline $\mathrm{SD}$ of $\Delta \mathrm{SDS}(1)$ & 0.19 & 0.21 & 0.25 & 0.29 & 0.33 \\
\hline Falling one band or more (\%) & 0.03 & 0.09 & 0.4 & 1.0 & 2.3 \\
\hline
\end{tabular}

curves on the British charts are $0.67 \mathrm{SD}$ score units apart, so a change of -0.38 unit is roughly half the distance between adjacent curves. Thus about $2 \%$ of reference children can be expected to fall by this amount between 5 and 6 years (and the same percentage to rise by the same amount over the same period).

Consider the height gain centile of a child whose height slips from the 50th to the 30th centile between 5 and 6 years - a fall in SD score of 0.5 units. So $\triangle \mathrm{SDS}=-0.5$, and from formula (2) the $\mathrm{SD}$ score for $\triangle \mathrm{SDS}$ is $-0.5 / 0.19=-2.6$ units, well below the 1 st centile. So this degree of centile crossing at this age is very uncommon.

The chance of $\triangle \mathrm{SDS}$ exceeding some cut off, say a fall of one centile band width between 5 and 6 years, can be calculated from formula (2). It gives the SD score corresponding to a one centile band width fall as $-0.67 / 0.19=$ -3.4 units, which is a chance of three in 10000 . So a fall from one centile curve to the next between 5 and 6 years of age is likely to be extremely rare.

\section{Discussion}

Changes in height centile over time can be interpreted using only the correlations in table 1. Although based on a group of French children measured during the 1950s and 1960 s, the correlations are similar to those from Tanner's Harpenden growth study, ${ }^{7}$ and are also likely to be valid for children now.

Routinely collected data tend to be of poorer quality than the research data on which the correlations of table 1 are based. To compensate for this, each correlation $r$ needs to be scaled down to $r^{\prime}$ using the formula:

$$
r^{\prime}=r\left\{1-\left[\frac{\delta^{2}-\varepsilon^{2}}{\sigma^{2}}\right]\right\}
$$

where $\sigma$ is the SD of height (about $5 \mathrm{~cm}$ ), $\varepsilon$ is the likely measurement error of height (that is the SD of a single measurement) in research studies (that for the French study is not known, but is likely to be between 0.1 and $0.4 \mathrm{~cm}$ - say $0.25 \mathrm{~cm}^{9}{ }^{10}$ ), and $\delta$ is the likely measurement error in routinely collected data (perhaps 0.8 $\mathrm{cm}$, although this is currently uncertain).

These figures reduce the correlation between 5 and 6 years from $r=0.981$ to $r^{\prime}=0.958$, which increases the SD of $\triangle$ SDS from 0.19 to 0.29 units, widens the centiles by $50 \%$, and dramatically increases the proportion of children falling one centile band width or more. Hindmarsh proposes a fall of one centile band width as the cut off for detecting low height gain. ${ }^{3}$ Table 2 shows the effect on this proportion of various sizes of measurement error, and it highlights the importance of keeping the measurement error as small as possible. At one extreme, with $\delta=0.25 \mathrm{~cm}$, where measurement error in the community matches that of research data, only three per 10000 children fall a whole centile band. At the other extreme, where the community measurement error is $1 \mathrm{~cm}$, the proportion falling one centile band is almost a hundredfold greater at $2.3 \%$. So Hindmarsh's proposal should screen in between three per 10000 and two per 100 subjects, depending on the quality of the measurements.

This uncertainty about the screening in rate is a fundamental problem of growth monitoring, as the effectiveness of the screening depends critically on knowing the size of the measurement error. Work in progress using routinely collected height data from the Child Health Computer System aims to estimate the size of the measurement error in the community setting, which will inform future plans for the routine measurement of height at ages beyond 5 years.

Until this information becomes available, table 3 gives the percentage of children likely to fall one centile band width between various ages based on an assumed community measurement error of $0.8 \mathrm{~cm}$. Voss et al have noted that most observers, measuring the same children repeatedly with the same instrument, achieve a measurement error of no more than $0.4 \mathrm{~cm} .{ }^{10}$ With several observers using different instruments the error is likely to be greater, and the chosen value is deliberately conservative. Tanner remarks that 'The standard error of height measurements made by an experienced anthropometrist is $0.2-0.3 \mathrm{~cm}$.... The errors of untrained, unspecialized and often uninterested school doctors and nurses can easily run to three time as much'. ${ }^{9}$ Note too that until 1995 the Child Health Computer System recorded height to the nearest whole centimetre, further inflating the measurement error. With better quality measurements the percentages in table 3 should be appreciably smaller. Continuous attention to quality control is the key.

If there are two or more successive periods of centile crossing, the percentages can be multiplied together (as successive velocities are essentially uncorrelated ${ }^{7}$ ). Take for example a child who falls one centile band width from 2 to 3 years ( $5 \%$ chance), then a further band width from 3 to 4 years ( $3 \%$ chance). The chance of 
Table 3 Percentage of children measured in the community falling one height centile band width or more over various time intervals

\begin{tabular}{|c|c|c|c|c|c|c|c|}
\hline & \multicolumn{7}{|c|}{ Age (years) } \\
\hline & 2 & 3 & 4 & 5 & 6 & 7 & 8 \\
\hline \multicolumn{8}{|c|}{ Age (years) } \\
\hline 3 & 5 & & & & & & \\
\hline 4 & 10 & 3 & & & & & \\
\hline 5 & 12 & 6 & 2 & & & & \\
\hline 6 & 14 & 8 & 3 & 1 & & & \\
\hline 7 & 14 & 9 & 5 & 2 & 1 & & \\
\hline 8 & 16 & 11 & 6 & 3 & 2 & 1 & \\
\hline 9 & 17 & 12 & 8 & 5 & 4 & 2 & 1 \\
\hline
\end{tabular}

Assumptions: community measurement error $\delta=0.8 \mathrm{~cm}$, research measurement error $\varepsilon=0.25$ $\mathrm{cm}$, population SD $\sigma=5 \mathrm{~cm}$. Example: from five to six years, $1 \%$ of children fall one centile band width or more. height measurements, the two formulas give very similar answers.

With weight and height, however, where the correlation is smaller, the difference between the two formulas is correspondingly greater. The weight-height difference is positively correlated with weight and negatively correlated with height, which makes short children appear fat and tall children thin. A better approach is to use the British body mass index reference, ${ }^{12}$ which is a conditional reference and is broadly unbiased with respect to height.

A final comment: centile crossing is defined here in terms of centile channels or bands, each band width corresponding to $0.67 \mathrm{SD}$ score units. Elsewhere it is sometimes defined as the number of centiles crossed, ${ }^{3}$ but this is ambiguous: a shift of about one centile band width may cross 0 , one or two centile curves. To avoid ambiguity, recommendations should be in terms of centile band width, not the number of centiles crossed.

\section{with the others, can be identified.}

If $\triangle$ SDS is based on weight and height measured at the same age, where the correlation is about 0.7 , the 2 nd and 98 th centiles for the weight-height difference are $\pm 2 \sqrt{2(1-0.7)}=$ \pm 1.5 SD score units, equal to two and a bit centile band widths difference between height and weight. The proposal of Hulse and Schilg of three centile bands is more conservative and corresponds to the 1 st and 99 th centiles. ${ }^{4}$ So $2 \%$ ( $1 \%$ fat and $1 \%$ thin) of children are likely to have a discrepancy of three centile band widths or more between their weight and height centiles.

Formula (1) is useful for comparing centiles derived from the British growth reference. However, it is based on the difference between centiles, which is not necessarily the best way to make the comparison, as it fails to adjust for regression to the mean. As a result, the centile difference $(\Delta S D S)$ tends to be negatively correlated with the first centile $\left(\operatorname{SDS}_{1}\right)$. An alternative is the conditional approach, ${ }^{11}$ which adjusts one measurement for the other and leads to a slightly different formula. If the correlation $r$ is close to 1 , as it is for successive I am grateful to David Hall, Peter Hindmarsh, Tony Hulse,
Linda Voss, and two anonymous referees for their comments and suggestions on earlier drafts of the paper. I also thank Michel Sempé and Marie-Françoise Rolland-Cachera for making available the French height data.

1 Hall DMB. Monitoring children's growth. BMF 1995;311:583-4.

2 Freeman JV, Cole TJ, Chinn S, Jones PRM, White EM, Preece MA. Cross sectional stature and weight reference curves for the UK, 1990. Arch Dis Child 1995;73:17-24.

3 Hindmarsh PC. Monitoring children's growth [letter]. BMf 1996;312:122.

4 Hulse JA, Schilg S. Monitoring children's growth [letter]. BMF 1996;312:122.

5 Sempé M, Pédron G, Roy-Pernot M. Auxologie: méthode et séquences. Paris: Theraplix, 1979.

6 Cole TJ. Growth charts for both cross-sectional and Cole TJ. Growth charts for both cross-sectic
longitudinal data. Stat Med 1994;13:2477-92.

7 Bailey BJR. Monitoring the heights of prepubertal children Bailey BJR. Monitoring the heights of prepubertal children
by the use of standard charts. Ann Hum Biol 1994;21:1-11. 8 Cole TJ. Weight/height ${ }^{p}$ compared to weight $/$ height $^{2}$ for age on p during puberty. Ann Hum Biol 1986;13:433-51.

9 Tanner JM. Physical development. Br Med Bull 1986;42: 131-8.

10 Voss LD, Bailey BJR, Cumming K, Wilkin TJ, Betts PR. The reliability of height measurement. Arch Dis Child 1990;65:1340-4

11 Cole TJ. Conditional reference charts to assess weight gain in British infants. Arch Dis Child 1995;73:8-16.

12 Cole TJ, Freeman JV, Preece MA. Body mass index reference curves for the UK, 1990. Arch Dis Child 1995;73: $25-9$. assessing adiposity in childhood: influence of age and bone 